\title{
Lung cancer screening: who pays? Who receives? The European perspectives
}

\author{
Pierluigi Novellis ${ }^{1}$, Silvia Raimondi Cominesi ${ }^{1}$, Francesca Rossetti ${ }^{1}$, Michele Mondoni ${ }^{2}$, Vanesa Gregorc ${ }^{3}$, \\ Giulia Veronesi ${ }^{1,4}$
}

${ }^{1}$ Division of Thoracic Surgery, IRCCS San Raffaele Scientific Institute, Milan, Italy; ${ }^{2}$ Department of Health Sciences, University of Milan, Respiratory Unit, ASST Santi Paolo e Carlo, Milan, Italy; ${ }^{3}$ Department of Medical Oncology, IRCCS San Raffaele Scientific Institute, Milan, Italy; ${ }^{4}$ Faculty of Medicine and Surgery, Vita-Salute San Raffaele University, Milan, Italy

Contributions: (I) Conception and design: G Veronesi; (II) Administrative Support: None; (III) Provision of study materials or patients: All authors; (IV) Collection and assembly of data: P Novellis, SR Cominesi, F Rossetti, G Veronesi; (V) Data analysis and interpretation: None; (VI) Manuscript writing: All authors; (VII) Final approval of manuscript: All authors.

Correspondence to: Pierluigi Novellis. Division of Thoracic Surgery, IRCCS San Raffaele Scientific Institute, Via Olgettina 58, 20132 Milan, Italy. Email: pierluigi.novellis84@gmail.com; novellis.pierluigi@hsr.it.

\begin{abstract}
Lung cancer is the leading cause of cancer-related death worldwide, and its early detection is critical to achieving a curative treatment and to reducing mortality. Low-dose computed tomography (LDCT) is a highly sensitive technique for detecting noninvasive small lung tumors in high-risk populations. We here analyze the current status of lung cancer screening (LCS) from a European point of view. With economic burden of health care in most European countries resting on the state, it is important to reduce costs of screening and improve its effectiveness. Current cost-effectiveness analyses on LCS have indicated a favorable economic profile. The most recently published analysis reported an incremental cost-effectiveness ratio (ICER) of €3,297 per 1 life-year gained adjusted for the quality of life (QALY) and €2,944 per life-year gained, demonstrating a $90 \%$ probability of ICER being below $€ 15,000$ and a $98.1 \%$ probability of being below $€ 25,000$. Different risk models have been used to identify the target population; among these, the PLCOM2012 in particular allows for the selection of the population to be screened with high sensitivity. Risk models should also be employed to define screening intervals, which can reduce the general number of LDCT scans after the baseline round. Future perspectives of screening in a European scenario are related to the will of the policy makers to implement policy on a large scale and to improve the effectiveness of a broad screening of smoking-related disease, including cardiovascular prevention, by measuring coronary calcium score on LDCT. The employment of artificial intelligence (AI) in imaging interpretation, the use of liquid biopsies for the characterization of CT-detected undetermined nodules, and less invasive, personalized surgical treatments, will improve the effectiveness of LCS.
\end{abstract}

Keywords: Lung cancer screening (LCS); low-dose computed tomography (LDCT); risk models; European LCS

Submitted May 22, 2020. Accepted for publication Apr 12, 2021.

doi: $10.21037 /$ tlcr-20-677

View this article at: http://dx.doi.org/10.21037/tlcr-20-677

\section{Introduction}

Lung cancer, which is the leading cause of cancer-related death worldwide, is responsible for 270,000 deaths yearly in Europe (1). Although the lung cancer mortality rate in men is projected to decline, in women it is predicted to rise, mainly due to the dissimilarity of gender-related smoking habits across generations, thus confirming the impact that cigarette smoking has on lung cancer in both sexes (2). Therefore, the first crucial step in lung cancer prevention should be smoking interruption, a point that is discussed with every single patient enrolled in lung cancer screening (LCS) protocols. 
Most lung cancer patients are diagnosed in an advanced stage, with an overall 5 -year survival of $18 \%$ (3). As the prognosis of lung cancer depends on tumor size and stage at diagnosis, early detection is critical to achieving a curative treatment and to reducing mortality.

It is well established that low-dose computed tomography (LDCT) is a highly sensitive technique for detecting noninvasive early lung tumors in the screening of high-risk populations (LCS) (4-13). Published in 2011, the large-sample, randomized National Lung Screening Trial (NLST) in the United States demonstrated that LDCT performed in highrisk patients reduces lung cancer mortality by more than $20 \%$ compared with groups screened by chest X-ray (13).

In Europe, several randomized screening trials comparing LDCT scan versus chest X-ray were released but had insufficient statistical power to demonstrate any mortality benefit (14). Recently, however, a largesample European randomized controlled trial, the DutchBelgian Randomized Lung Cancer Screening Trial (NELSON study) reported that the 10-year lung cancer mortality in the LDCT screening group was 2.5 deaths per 1,000 person-years, while that in the control group was 3.30 . The screening arm therefore showed a $24 \%$ lung cancer mortality reduction (cumulative incidence ratio for death from lung cancer of $0.76 ; \mathrm{P}=0.01$ ). It should be noted that in the screening arm, about $77.6 \%$ of diagnosed cancers were early stage (IA to II), while $71.6 \%$ of patients in the control arm were stage III to IV at diagnosis, indicating that earlystage diagnosis leads to a reduction in mortality (15).

Other European single-arm studies have analyzed oncological outcomes. In Milan for instance, the Continuous Observation of Smoking Subjects (COSMOS), a single-center nonrandomized LDCT screening study, showed high sensitivity (90.3\%) and specificity (99.4\%) for the first 5 years of follow-up and confirmed that $78 \%$ of detected tumors were localized (N0 M0); a high proportion $(87 \%)$ of cancers were radically treated, and long-term oncological results were satisfactory with an overall 5 -year survival of $78 \%$ (16).

\section{Potential drawbacks}

Despite its widely recognized value, LDCT screening in Europe has to overcome several obstacles in its implementation, especially regarding its economic impact (which is discussed below), ionizing radiation exposure, overdiagnosis (defined as the detection of a lung cancer that would not lead to patient's death because of a slow growth rate and competing age-related risks for death), and false positives.

According to the retrospective evaluation in the 10-year COSMOS study, cumulative radiation exposure and a lifetime risk of radiation-induced cancer can be acceptable if we consider the substantial mortality reduction associated with LCS; in fact, in a worst case scenario, only 1 radiationinduced cancer would be expected in every 108 lung cancers detected after 10 years of LDCT-LCS. Nevertheless, it would be possible to further reduce exposure by improving the diagnostic flowchart and excluding low-risk patients to minimize unnecessary radiologic examinations (17). Furthermore, new CT scanners with optimized acquisition protocols that can reduce the dose by up to $40 \%$ and new reconstruction algorithms that can reduce the radiation dose by up to $80 \%$ and obtain an equivalent image quality are now available (18-20).

Some authors consider LDCT screening of limited utility in changing the natural history of lung cancer due to overdiagnosis (21-23) of indolent early-stage cancers considered of uncertain clinical relevance (23). Together with the potentially high false-positive rate, overdiagnosis is one of the most criticized drawbacks of LDCT screening. A retrospective analysis on the COSMOS population suggests that changes in size on sequential LDCT screening can help in the discrimination of aggressive versus indolent tumors by measuring the volume-doubling time (VDT) to classify them as fast growing ( $<400$ days), slow growing (400 to 599 days), or indolent ( $>600$ days). VDT seems to be an acceptable indicator of cancer aggressiveness and its analysis shows that only fewer than $10 \%$ of resected screened lung cancers have a VDT higher than 600, thus minimizing the problem of overdiagnosis. As this $10 \%$ of indolent cancers may include some of the overdiagnosed cancers and nonsurgical treatments, such as stereotactic ablative radiotherapy (SABR) or minimally invasive (video-assisted/robot-assisted), limited resections should be considered to reduce overtreatment (24). In the ITALUNG trial, a randomized controlled trial carried out in Tuscany from 2004 comparing LDCT-LCS to standard care, the number of cancer cases diagnosed was similar in the two arms, showing no overdiagnosis after an adequate follow-up period (25).

As far as false positives are concerned, during the first 6 years (from 2004-2005 to 2010) of the COSMOS study, only $14 \%$ of benign lesions underwent surgical biopsy (24) due to the application of positron emission tomography-CT (PET-CT) for preoperative assessment of indeterminate screening-detected lung nodules and the repetition of 
LDCT after 1 month in case of suspected inflammatory lesions (26). Even if the nodules had typical benign characteristics, half of them were PET-positive and half of them had a VTD in the rate of malignancy, and thus surgery was performed; fine-needle aspiration biopsy (FNAB) was not routinely used in this screening protocol. This percentage is lower than that reported in the NLST, where $25 \%$ of suspicious cases underwent invasive procedures for benign nodules (27), and that in the NELSON study, where $27 \%$ and $21 \%$ of cases underwent surgery for benign disease at baseline and in subsequent rounds, respectively (12). By contrast, the Danish LCS Trial reported that only $10 \%$ of benign cases underwent surgery using a protocol that combined PET-CT with VDT (28). Improved diagnostic protocols, a prolonged period of follow-up, and a multidisciplinary approach are critical to reducing invasive procedures for benign nodules that often cause unnecessary morbidity and resource utilization. Screening is therefore an important tool for reducing deaths from lung cancer and can be considered effective and safe.

In this review we explore some aspects of the screening process, particularly those regarding the cost-effectiveness analysis (CEA) and the optimization of target population selection and recruitment.

\section{CEA in the European and International setting}

It is necessary to demonstrate that LDCT-LCS can be costeffectively implemented. LCS costs were initially (from 2000 to 2007) a predominantly North American interest, and early studies were published in the USA and Canada (29,30-42). A CEA based on NLST data, which compared an LDCT screening arm with a standard care arm in over 53,000 individuals, estimated a cost of USD $\$ 81,000$ per quality-adjusted life-year (QALY) gained (30-36). Thus, the US Preventive Services Task Force (USPSTF) recommended screening for high-risk individuals (aged 55 to 80 years, with at least 30 pack-years smoking history and fewer than 15 years of smoking abstinence immediately before screening) (43), with private and public institutions covering costs. In Canada, a group conducted a CEA comparing patients undergoing LDCT against nonscreened individuals: the high-risk screening cost was estimated to be CAD \$20,724 (in 2015) per QALY gained $(34,35)$.

Nevertheless, it is difficult to apply these results to the European framework, where costs and reimbursements are markedly different from those in North America. Only recently (from 2014 to 2019), CEAs have been published in Europe (44-49). Two studies published in Germany examined the incremental cost-effectiveness ratio (ICER): one reported $€ 19,302$ per life-year gained and €30,241 per QALY and the other reported $€ 16,754-23,847$ per life-year $(45,46)$. In the UK, two studies found an ICER lower than $£ 11,000$ per QALY $(44,47)$; a more recent study found an ICER between $£ 20,000$ and $\$ 30,000$ per QALY, but under extreme assumptions (very small cost advantage in treating stage I and stage IV, no increase in average survival between the screening and nonscreening scenarios) (48). Meanwhile, a Polish study reported an ICER of $€ 1,353$ per life-year gained (49).

For Italy, our group found an ICER of $€ 3,297$ per QALY gained and €2,944 per life-year gained (50). This is the most recent study covering this issue and included the new treatment options of advanced stage lung cancer. This model is based on a decision tree presenting the main contributors to costs, with each branch representing a divergence in the management of the screened patient; usual care arms and the alternatives were assigned probabilities of occurrence. Real-world cost data were estimated by summing all reimbursements paid to the hospital for hospitalizations, outpatient appointments, examinations, and medications, sorted by year of diagnosis and disease stage. The screening arm was composed of individuals enrolled in the COSMOS study (51) who represented a high-risk population, adapted by considering only those who met the more stringent eligibility criteria of the USPSTF. Patients diagnosed and treated for lung cancer in the Lombardy region of Italy constituted the nonscreening arm. Data on a sample of subjects surveyed in 2015 (52) were extrapolated to estimate individuals at high risk of lung cancer in Italy in 2017: the Italian National Institute of Statistics (ISTAT) (53) estimated a population of $17,757,163$ people aged $55-80$ years old in 2017, including 2,166,374 individuals at high risk of lung cancer. Based on the obtained ICERs, screening this population for 5 years would require an investment by the Italian Health Service of around $€ 600$ million at $€ 120$ million/year, which represents $2.4 \%$ of the yearly Italian budget for disease prevention ( $€ 5$ billion). Further investigation of sensitivity using Monte Carlo simulations demonstrated a $55 \%$ probability of the ICER per life-year being less than $€ 5,000$, an $80 \%$ probability of being less than $€ 10,000$, a $90 \%$ probability of being less than $€ 15,000$, and a $98.1 \%$ probability of being less than the threshold of $€ 25,000$, as indicated by the UK's the National Institute for Health and Care Excellence (NICE) introduction for new medical technologies (54). These low ICERs imply that 
screening can be implemented throughout Italy at relatively low cost, thereby cost-effectively saving the lives of many lung cancer patients.

The recent introduction of new targeted therapies with monoclonal antibodies and immunotherapy in the treatment of late-stage lung cancer has changed the natural history of mutation-bearing diseases. Despite their undeniable efficacy, these treatments are extremely expensive: osimertinib treatment, for example, could be more effective than chemotherapy in advanced lung cancer patients with an acquired epidermal growth factor receptor (EGFR) T790M mutation; nevertheless, this benefit has been associated with a significant cost increase, with average ICERs greater than US \$200,000 per QALY in the United States and greater than US \$30,000 per QALY in China. Considering the threshold of US $\$ 100,000$ and US $\$ 23,815$ per QALY gained in the USA and in China, respectively, osimertinib was not considered a cost-effective therapeutic strategy (55); now, however, owing to its associated survival advantage, osimertinib has been indicated as first-line therapy in the treatment of non-small cell lung cancer (NSCLC) since 2018. The capability of LCS to identify lung cancer in the early stages may decrease the total burden of overall lung cancer treatment by reducing the number of patients diagnosed at a late stage and thus those requiring molecular profile identification and expensive targeted therapies.

\section{Selection of target population and screening interval: risk models}

European healthcare systems are mainly nationalized, with charges being covered by governments. Costs are probably one of the factors that have limited the introduction of LCS into clinical practice. The use of different risk models can have a dual purpose: for one, it allows for the identification of subjects at higher risk of developing lung cancer; for another, it can create a tailored screening interval profile useful for designing a more personalized and cost-effective screening schedule. The NLST recruitment of subjects was based on pack-years $\geq 30$, quit-time in former smokers of $\leq 15$ years, and age 55 to 74 years. The USPSTF and the Centers for Medicare and Medicaid Services (CMS) recommendations applied the same criteria as the NLST, but used the ages of 55-80 and 55-77 years, respectively $(43,56)$. The risk model associated with NLST has the power to identify 1 tumor out of 320 screened subjects (57). These values are difficult to apply in a European scenario.

LCS recruitment is based on smoking status and age.
Assuming that age and cigarette smoking are the most important but not the sole risk factors for lung cancer development and considering the need for a more powerful system to select people at high risk of lung cancer, several risk models have been proposed over the years $(51,58-76)$. However, many of them have been considered unsuitable, as they were validated in restricted or non-European populations or have only modest predictive power (59,60,62-68,70,71,73-76).

The Prostate, Lung, Colorectal, and Ovarian (PLCOm2012) Cancer Screening Trial (69) was thus remade in 2012 to estimate cancer development risk over 6 years. The model was built on a cohort of 80,375 individuals and with an external validation of 37,332 subjects. The PLCOm2012 prediction rules were evaluated at a $1.34 \%$ risk threshold. The PLCOm2012 model was demonstrated to be more successful than the NLST criteria: "sensitivities were $83.0 \%$ versus $71.1 \%(P<0.001)$, the specificities were $62.7 \%$ versus $62.9 \%(P=0.54)$, and the positive predictive values were $4.0 \%$ versus $3.4 \%(P=0.01)$ " (72). The model in fact identified 81 more cancers more than the NLST did.

Similarly, the Liverpool Lung Project (LLP) model was used to calculate the 5 -year lung cancer risk in a population aged 40 to 80 years. The model was designed using 1,736 case-control-matched individuals in a population from Liverpool, UK. The LLP model was used in the United Kingdom Lung Screening trial. The trial used LDCT for the early detection of lung cancer in highrisk subjects ( $>5 \%$ risk over 5 years, according to the LLP model) aged 50 to 75 years (77). The high-risk threshold may be a cost-effective method, as it excludes a high proportion of controls, but it will, however, only capture a small proportion of cases. The United Kingdom Lung Screening uses a single screen rather than multiple screens of the identified individuals. This is done to maximize the yield of the screening test, making it more cost-effective (78). Recently, the NHS England Lung Health Projects (79) has been using both the PLCOM2012 and the LLP v.2 model, with the thresholds adopted for screening the eligibility of PLCOM2012 being $\geq 1.3 \%, \geq 1.5 \%$, and $\geq 2.0 \%$.

In order to improve screening and limit its costs, in 2011, we developed a risk model to optimize the interval between the screening rounds according to individual risk. We stratified the population of the COSMOS study integrating characteristics of the subjects (i.e., age, gender, smoking habit) with findings of baseline CT scans (emphysema and features of nodules) (51). Presence of nonsolid nodules [risk ratio (RR): 10.1, 95\% confidence interval (CI): 5.57-18.5], 


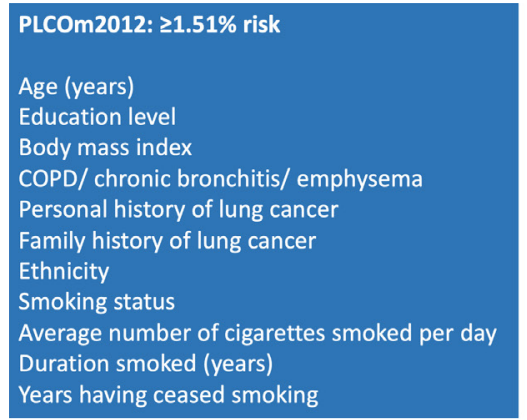

SCREENING INTERVAL

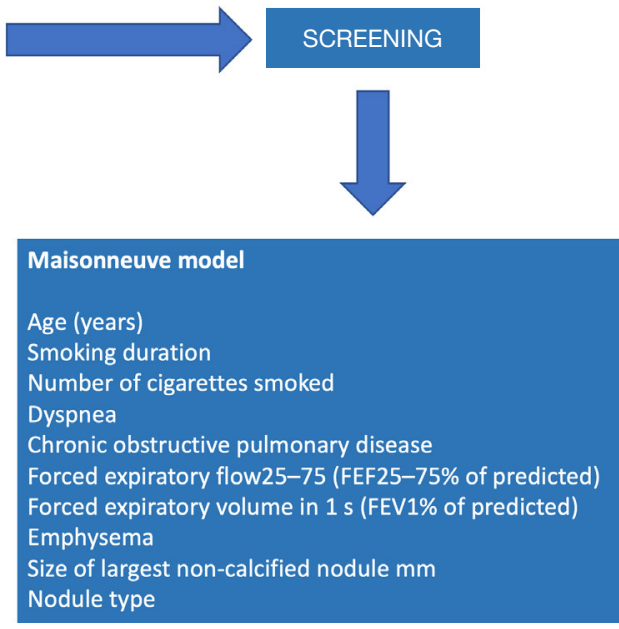

Figure 1 In this schematic model, the current SMAC screening strategy is reapplied. We identify subjects to be screened using the PLCOm2012 risk model to select the population at high risk of developing lung cancer. After the first CT scan, a second risk (Maisonneuve model) model is used to determine the correct interval to perform the second round of CT in order to improve the effectiveness of the screening.

nodule size more than $8 \mathrm{~mm}$ (RR: 9.89, 95\% CI: 5.84-16.8), and emphysema (RR: 2.36, 95\% CI: 1.59-3.49) at baseline CT were all significant predictors of malignity.

The PLCOm2012 risk model associated with the Maisonneuve model that integrates CT findings at baseline screening could help in both the selection of target population and the definition of the optimal interval of screening. The Italian SMAC study represents an external validation of this model. Other studies have been aimed in this direction: in 2019, Pastorino et al. presented the results of a risk model to the World Conference on Lung Cancer (80); they reported that baseline blood microRNA associated with LDCT could predict lung cancer risk and mortality at 4 years and that lung cancer risk was significantly higher in subjects with a single-positive [hazard ratio (HR): 6] and double-positive test (HR: 36.6). According to this model, blood microRNA plus LDCT would allow optimal screening intensity and reduce unnecessary repetition of LDCT, thus improving the efficacy of screening (80) (Figure 1).

\section{Future perspectives}

Many aspects of LCS have been overlooked over the years to improve its cost-effectiveness. The future perspectives of LCS in Europe will involve several aspects. First, as LCS addresses the smoking population, LCS may be applied to the screening of other smoking-related diseases, including both cardiovascular and pulmonary disorders [e.g., emphysema/chronic obstructive pulmonary disease (COPD) and idiopathic pulmonary fibrosis (IPF)] $(81,82)$. Notably, an early diagnosis of IPF might induce an early prescription of antifibrotic therapy with beneficial effects on survival (83).

Secondly, as LCS allows for the detection of more tumors at early stages, there should be a subsequent increase in the number of minimally invasive parenchyma-sparing surgical procedures. Other objectives could be the improvement of the CT reading process using artificial intelligence (AI) software and the search for biomarkers to facilitate speedier diagnoses without the need of invasive procedures.

\section{Prevention and detection of cardiovascular diseases (CVD)}

CVD were reported to be responsible for the majority of total deaths in LDCT study arms (13). Coronary artery calcification (CAC), extracoronary cardiac calcifications, and aortic aneurysm can all be detected on LDCT scans.

LCS gives offers the attractive opportunity to reduce CVD mortality through the concomitant assessment of CVD risk via coronary artery calcium quantification, a recognized marker of atherosclerosis (84).

CAC alone, however, is not sufficient for evaluating CVD risk (85). A recent consensus paper reported that 

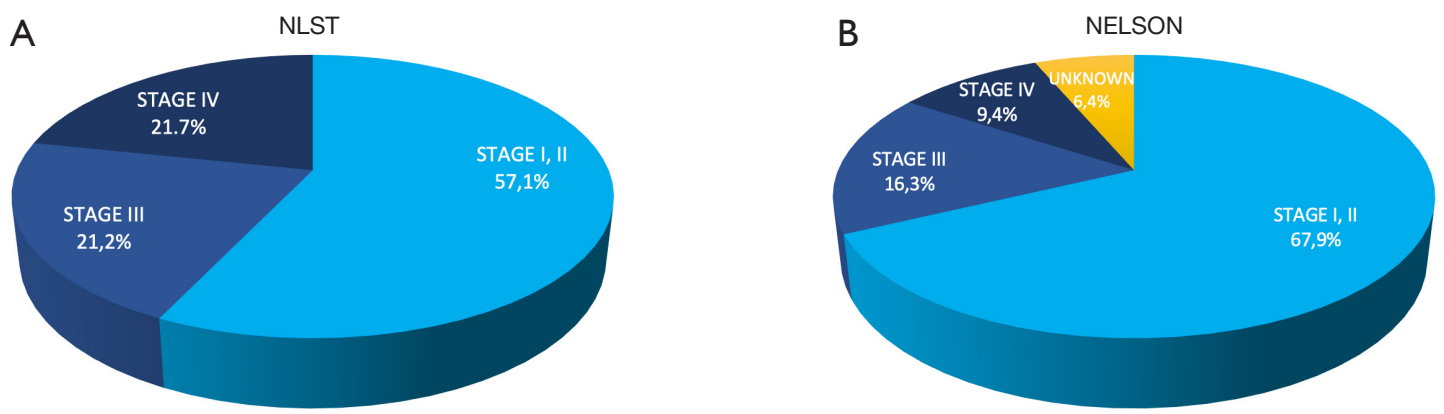

cosmos

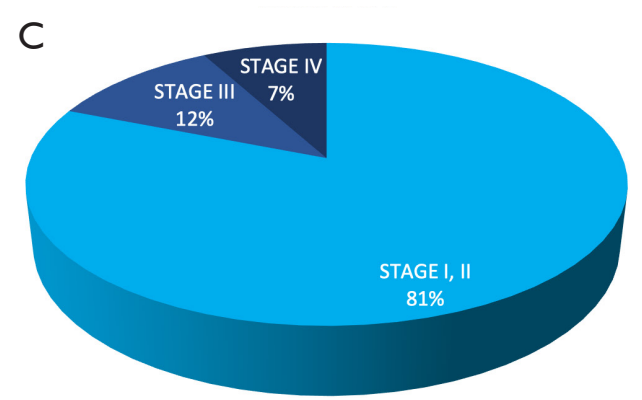

Figure 2 Lung cancer stage distribution in different randomized LDCT screening [NLST in (A), Nelson in (B)], and nonrandomized studies [COSMOS in (C)].

CAC on a basal CT associated to the Framingham score can refine risk stratification (86). The NICE guidelines recommend the use of statin (atorvastatin $20 \mathrm{mg}$ ) in the case of poor control of cholesterol values associated with CAC expression (QRISK $2 \geq 10 \%$ ) (87). The QRISK 2 is an algorithm developed by the UK National Health Service that calculates the risk of heart attack or stroke over the ensuing 10 years (87).

In 2019, Ruparel et al.'s study demonstrated the following phenomena: (I) in a cohort of individuals undergoing an LDCT for LCS, $62 \%$ of participants had coronary calcium; (II) an increased QRISK 2 was associated with s higher value of CAC on LDCT; and (III) $98 \%$ of LCS-eligible individuals met the $\geq 10 \% 10$-year CVD risk threshold required for statin primary prevention of CVD events in the UK (88). LDCT could therefore not only serve an important role in secondary prevention for lung cancer but also aid in the primary prevention of CVD.

\section{Increase in minimally invasive surgical procedures}

The main goal of LCS is to reduce mortality. However, to increase the effectiveness of LCS, other treatments should to be adapted and improved upon. Specifically, because of the increasing number of small, early-stage tumors detected by LCS, there should be a concurrent increase in the number of minimally invasive procedures that are associated with better and shorter postoperative hospitalizations and reduced costs.

Surgical results of the NLST indicated that $80 \%$ of patients received lobectomy, $4.1 \%$ pneumonectomy, and $16.1 \%$ sublobar resection ( $69 \%$ wedge resection), while only $29.6 \%$ $(n=305)$ of the cohort had a thoracoscopic resection (89).

In the Danish LCS trial, $84 \%$ of surgical procedures on screen-detected cancers were performed by video-assisted thoracic surgery (90). This high number of minimally invasive procedures was not reported in other screening programs $(91,92)$. Similarly, in the SMAC study, we treated the majority of cancers using a minimally invasive approach, specifically, a robot-assisted technique.

Abundant evidence supports the superiority of minimally invasive surgery of thoracotomy in terms of perioperative and oncological outcomes and postoperative quality of life and pain control (93-96). However, few data have been reported for anatomic sublobar resections for tumors diagnosed by screening. In the NLST, only $5 \%$ of resections were anatomic segmentectomies (89), while in the Danish LCS trial, this figure was only 2\% (90) (Figure 2). Nevertheless, the number of anatomic segmentectomies is expected to increase in the future. 
In recent years, robotic surgery has shown benefits in reducing mortality, shortening length of stay, and decreasing the severity of hospitalization (97). Moreover, it has been associated with a lower conversion-to-open rate and a lower 30-day complication rate (98). Robotic surgery has also demonstrated advantages in the field of segmentectomy (99-103). Robotic segmentectomies seem to be characterized by absence of major bleeding, an "oncologically acceptable" number of lymph nodes removed, $0 \%$ mortalities, and only 3.4\% 30-month local recurrence (100). Robotic segmentectomies also show precise identification of the intersegmental plane in $100 \%$ of cases, thus facilitating radical resection (104). Minimally invasive sublobar resections could be the standard for slow-growing and small tumors detected at screening.

\section{AI for radiological analysis}

If screening is covered by the NHS, the number of LDCT scans will greatly increase. Guidelines today help in therapeutic decision-making when a solitary lung nodule is detected $(105,106)$; however, the radiological reporting of the exams requires a significant amount of medical expertise and labor. New software based on AI can aid in analyzing nodule size, margins, attenuation, and other radiological features without the subjective judgement of the radiologist. In the UK, the IDEAL study (Artificial Intelligence and Big Data for Early Lung Cancer Diagnosis), developed by Optellum Ltd., used AI to evaluate the probability of a lung nodule to be malignant. As a risk prediction model, the lung cancer prediction convolutional neural network (LCP-CNN) was constructed using a machine learning algorithm (107). Recently Baldwin (108) and colleagues compared the performance of the LCP-CNN with the Brock University model recommended in UK guidelines. Their results showed an area under curve of $89.6 \%$ for LCP-CNN (95\% CI: 87.6-91.5), compared with $86.8 \%$ (95\% CI: 84.3-89.1) of the Brock model $(\mathrm{P} \leq 0.005)$. The LCP-CNN score provided better discrimination, with a larger proportion of benign nodules identified without missing cancers, thus reducing the proportion of follow-up CT scans and saving resources. As AI technology progresses, CT reports will serve as their own validation, making the $\mathrm{CT}$ screening faster and more costeffective.

\section{Liquid biopsies for preoperative diagnosis}

The potential risk related to the rising number of lung nodules detected by LDCT may translate into an increase in invasive diagnostic procedures and diagnostic surgery. Liquid biopsy may be the solution to this issue. Circulating exosomes (109), microRNA (110), cell-free DNA (cfDNA), and circulating tumor cells (CTCs) are being evaluated for their clinical utility (111). Thus far, research into cfDNAs and CTCs has yielded the most promising results. cfDNA is the portion of circulating DNA in bloodstream that is passively released from apoptotic and necrotic cells or indirectly by tumor-associated macrophages. Migration of CTCs in the bloodstream is an early event in carcinogenesis: they are released by cancer tissue as single cells or clusters of cells that can be isolated in the peripheral blood in different ways (111). Ilie et al. examined the presence of "sentinel" CTCs in COPD patients without radiologically detectable lung cancer and screened them annually with LDCT: CTCs were detected in $3 \%$ of COPD patients, and, in 1 to 4 years, these patients developed a radiologically detectable lung nodule, leading to prompt surgical intervention of an earlystage tumor (112).

These results support the use of liquid biopsies as a tool for noninvasive preoperative diagnosis of lung cancers in the LCS setting, which can reduce the number of invasive diagnostic procedures, such as endobronchial ultrasoundguided transbronchial needle aspiration, CT-guided biopsies and diagnostic surgeries, and improve the effectiveness of the treatment by reducing the time required for these preoperative invasive diagnostic procedures.

\section{Conclusions}

It is well established that the application of LCS can reduce mortality, justifying its adoption by European public healthcare systems. This, however, strictly necessitates that LCS be rendered as cost-effective as possible. To improve effectiveness, risk models should be used according to the individual risk of the subjects both for the selection of the screening population and for the identification of optimal screening round intervals. This would provide suitable subjects for screening and individually tailored screening intervals.

CVD are the major cause of morbidity and mortality in populations eligible for screening: as the risk factors between lung cancer and CVD overlap, LCS could play an important role in prevention of smoking-related pathologies.

Finally, the use of AI in the interpretation of CT images and liquid biopsies for the histological and molecular 
definition of neoplasms could further improve the effectiveness of screening.

\section{Acknowledgments}

Funding: None.

\section{Footnote}

Provenance and Peer Review: This article was commissioned by the guest editors (Paul Van Schil and Annemiek Snoeckx) for the series "Lung cancer screening" published in Translational Lung Cancer Research. The article has undergone external peer review.

Peer Review File: Available at http://dx.doi.org/10.21037/ tlcr-20-677

Conflicts of Interest: All authors have completed the ICMJE uniform disclosure form (available at http://dx.doi. org/10.21037/tlcr-20-677). The series "Lung cancer screening" was commissioned by the editorial office without any funding or sponsorship. PN reports grants from Umberto Veronesi Foundation (FUV). GV reports grants from Associazione Italiana per la Ricerca sul Cancro (AIRC), Ministry of Health, Istituto Nazionale Assicurazione Infortuni sul Lavoro; outside the submitted work, she has received honoraria from $\mathrm{Ab}$ Medica $\mathrm{SpA}$. The authors have no other conflicts of interest to declare.

Ethical Statement: The authors are accountable for all aspects of the work in ensuring that questions related to the accuracy or integrity of any part of the work are appropriately investigated and resolved.

Open Access Statement: This is an Open Access article distributed in accordance with the Creative Commons Attribution-NonCommercial-NoDerivs 4.0 International License (CC BY-NC-ND 4.0), which permits the noncommercial replication and distribution of the article with the strict proviso that no changes or edits are made and the original work is properly cited (including links to both the formal publication through the relevant DOI and the license). See: https://creativecommons.org/licenses/by-nc-nd/4.0/.

\section{References}

1. Oudkerk M, Devaraj A, Vliegenthart R, et al. European position statement on lung cancer screening. Lancet Oncol 2017;18:e754-66.

2. Malvezzi M, Carioli G, Bertuccio P, et al. European cancer mortality predictions for the year 2017, with focus on lung cancer. Ann Oncol 2017;28:1117-23.

3. Siegel RL, Miller KD, Jemal A. Cancer statistics, 2019. CA Cancer J Clin 2019;69:7-34.

4. Henschke CI, McCauley DI, Yankelevitz DF, et al. Early Lung Cancer Action Project: overall design and findings from baseline screening. Lancet 1999;354:99-105.

5. Diederich S, Thomas M, Semik M, et al. Screening for early lung cancer with low-dose spiral computed tomography: results of annual follow-up examinations in asymptomatic smokers. Eur Radiol 2004;14:691-702.

6. Sone S, Li F, Yang ZG, et al. Characteristics of small lung cancers invisible on conventional chest radiography and detected by population based screening using spiral CT. Br J Radiol 2000;73:137-45.

7. Swensen SJ, Jett JR, Hartman TE, et al. Lung cancer screening with CT: Mayo Clinic experience. Radiology 2003;226:756-61.

8. Pelosi G, Sonzogni A, Veronesi G, et al. Pathologic and molecular features of screening low-dose computed tomography (LDCT)-detected lung cancer: a baseline and 2-year repeat study. Lung Cancer 2008;62:202-14.

9. Mulshine JL, Sullivan DC. Clinical practice. Lung cancer screening. N Engl J Med 2005;352:2714-20.

10. Veronesi G, Bellomi M, Mulshine JL, et al. Lung cancer screening with low-dose computed tomography: a noninvasive diagnostic protocol for baseline lung nodules. Lung Cancer 2008;61:340-9.

11. Veronesi G, Bellomi M, Scanagatta P, et al. Difficulties encountered managing nodules detected during a computed tomography lung cancer screening program. J Thorac Cardiovasc Surg 2008;136:611-7.

12. van Klaveren RJ, Oudkerk M, Prokop M, et al. Management of lung nodules detected by volume CT scanning. N Engl J Med 2009;361:2221-9.

13. Aberle DR, Adams AM, Berg CD, et al. Reduced lungcancer mortality with low-dose computed tomographic screening. N Engl J Med 2011;365:395-409.

14. Veronesi G. Lung cancer screening: the European perspective. Thorac Surg Clin 2015;25:161-74.

15. de Koning HJ, van der Aalst CM, de Jong PA, et al. Reduced lung-cancer mortality with volume CT screening in a randomized trial. N Engl J Med 2020;382:503-13.

16. Veronesi G, Maisonneuve P, Spaggiari L, et al. Diagnostic performance of low-dose computed tomography 
screening for lung cancer over five years. J Thorac Oncol 2014;9:935-9.

17. Rampinelli C, De Marco P, Origgi D, et al. Exposure to low dose computed tomography for lung cancer screening and risk of cancer: secondary analysis of trial data and riskbenefit analysis. BMJ 2017;356:j347.

18. Yamada Y, Jinzaki M, Tanami Y, et al. Model-based iterative reconstruction technique for ultralow-dose computed tomography of the lung: a pilot study. Invest Radiol 2012;47:482-9.

19. Mathieu KB, Ai H, Fox PS, et al. Radiation dose reduction for CT lung cancer screening using ASIR and MBIR: a phantom study. J Appl Clin Med Phys 2014;15:4515.

20. den Harder AM, Willemink MJ, de Ruiter QMB, et al. Achievable dose reduction using iterative reconstruction for chest computed tomography: a systematic review. Eur J Radiol 2015;84:2307-13.

21. Welch HG, Black WC. Overdiagnosis in cancer. J Natl Cancer Inst 2010;102:605-13.

22. Fontana RS, Sanderson DR, Woolner LB, et al. Screening for lung cancer. A critique of the Mayo Lung Project. Cancer 1991;67:1155-64.

23. Bach PB. Is our natural-history model of lung cancer wrong? Lancet Oncol 2008;9:693-7.

24. Veronesi G, Maisonneuve P, Bellomi M, et al. Estimating overdiagnosis in low-dose computed tomography screening for lung cancer: a cohort study. Ann Intern Med 2012;157:776-84.

25. Paci E, Puliti D, Lopes Pegna A, et al. Mortality, survival and incidence rates in the ITALUNG randomised lung cancer screening trial. Thorax 2017;72:825-31.

26. Veronesi G, Travaini LL, Maisonneuve P, et al. Positron emission tomography in the diagnostic workup of screening-detected lung nodules. Eur Respir J 2015;45:501-10.

27. Aberle DR, DeMello S, Berg CD, et al. Results of the two incidence screenings in the National Lung Screening Trial. N Engl J Med 2013;369:920-31.

28. Ashraf H, Dirksen A, Loft A, et al. Combined use of positron emission tomography and volume doubling time in lung cancer screening with low-dose CT scanning. Thorax 2011;66:315-9.

29. Black WC, Gareen IF, Soneji SS, et al. Cost-effectiveness of CT screening in the National Lung Screening Trial. N Engl J Med 2014;371:1793-802.

30. Black WC. Computed tomography screening for lung cancer in the National Lung Screening Trial: a costeffectiveness analysis. J Thorac Imaging 2015;30:79-87.
Erratum in: J Thorac Imaging. 2015;30:W59.

31. Beinfeld MT, Wittenberg E, Gazelle GS. Costeffectiveness of whole-body CT screening. Radiology 2005;234:415-22.

32. Manser R, Dalton A, Carter R, et al. Cost-effectiveness analysis of screening for lung cancer with low dose spiral CT (computed tomography) in the Australian setting. Lung Cancer 2005;48:171-85.

33. McMahon PM, Kong CY, Bouzan C, et al. Costeffectiveness of computed tomography screening for lung cancer in the United States. J Thorac Oncol 2011;6:1841-8.

34. Villanti AC, Jiang Y, Abrams DB, et al. A cost-utility analysis of lung cancer screening and the additional benefits of incorporating smoking cessation interventions. PLoS One 2013;8:e71379.

35. Pyenson BS, Henschke CI, Yankelevitz DF, et al. Offering lung cancer screening to high-risk medicare beneficiaries saves lives and is cost-effective: an actuarial analysis. Am Health Drug Benefits 2014;7:272-82.

36. Raymakers AJN, Mayo J, Lam S, et al. Cost-effectiveness analyses of lung cancer screening strategies using low-dose computed tomography: a systematic review. Appl Health Econ Health Policy 2016;14:409-18.

37. Pyenson BS, Sander MS, Jiang Y, et al. An actuarial analysis shows that offering lung cancer screening as an insurance benefit would save lives at relatively low cost. Health Aff (Millwood) 2012;31:770-9.

38. Cressman S, Peacock SJ, Tammemägi MC, et al. The costeffectiveness of high-risk lung cancer screening and drivers of program efficiency. J Thorac Oncol 2017;12:1210-22.

39. Cressman S, Lam S, Tammemagi MC, et al. Resource utilization and costs during the initial years of lung cancer screening with computed tomography in Canada. J Thorac Oncol 2014;9:1449-58.

40. Shmueli A, Fraifeld S, Peretz T, et al. Cost-effectiveness of baseline low-dose computed tomography screening for lung cancer: the Israeli experience. Value Health 2013;16:922-31.

41. Marshall D, Simpson KN, Earle CC, et al. Economic decision analysis model of screening for lung cancer. Eur J Cancer 2001;37:1759-67.

42. Chirikos TN, Hazelton T, Tockman M, Clark R. Cost-effectiveness of screening for lung cancer. JAMA 2003;289:2358; author reply 2358-9.

43. Moyer VA; U.S. Preventive Services Task Force. Screening for lung cancer: U.S. Preventive Services Task Force recommendation statement. Ann Intern Med 
2014;160:330-8.

44. Field JK, Duffy SW, Baldwin DR, et al. The UK Lung Cancer Screening Trial: a pilot randomised controlled trial of low-dose computed tomography screening for the early detection of lung cancer. Health Technol Assess 2016;20:1-146.

45. Hofer F, Kauczor HU, Stargardt T. Cost-utility analysis of a potential lung cancer screening program for a highrisk population in Germany: A MOdelling approach. Lung Cancer 2018;124:189-98.

46. Treskova M, Aumann I, Golpon H, et al. Trade-off between benefits, harms and economic efficiency of lowdose CT lung cancer screening: a microsimulation analysis of nodule management strategies in a population-based setting. BMC Med 2017;15:162.

47. Hinde S, Crilly T, Balata H, et al. The cost-effectiveness of the Manchester "lung health checks", a community-based lung cancer low-dose CT screening pilot. Lung Cancer 2018;126:119-24.

48. Snowsill T, Yang H, Griffin E, et al. Low-dose computed tomography for lung cancer screening in high-risk populations: a systematic review and economic evaluation. Health Technol Assess 2018;22:1-276.

49. Kanarkiewicz M, Szcz sny TJ, Krysi ski J, et al. Costeffectiveness analysis of lung cancer screening with lowdose computerised tomography of the chest in Poland. Contemp Oncol (Pozn) 2015;19:480-6.

50. Veronesi G, Navone N, Novellis P, et al. Favorable incremental cost-effectiveness ratio for lung cancer screening in Italy. Lung Cancer 2020;143:73-9.

51. Maisonneuve P, Bagnardi V, Bellomi M, et al. Lung cancer risk prediction to select smokers for screening CT--a model based on the Italian COSMOS trial. Cancer Prev Res (Phila) 2011;4:1778-89.

52. Lugo A, Zuccaro P, Pacifici R, et al. Smoking in Italy in 2015-2016: prevalence, trends, roll-your-own cigarettes, and attitudes towards incoming regulations. Tumori 2017;103:353-9.

53. Available online: http://demo.istat.it/str2017/index.html

54. Guide to the methods of technology appraisal 2013. Available online: https://www.nice.org.uk/process/pmg9

55. Wu B, Gu X, Zhang Q. Cost-effectiveness of osimertinib for EGFR mutation-positive non-small cell lung cancer after progression following first-line EGFR TKI therapy. J Thorac Oncol 2018;13:184-93.

56. Decision memo for screening for lung cancer with low dose computed tomography (LDCT) (CAG-00439N). Available online: https://www.cms.gov/medicare-coverage-
database/details/nca-decision-memo.aspx?NCAId=274\&N

57. Tota JE, Ramanakumar AV, Franco EL. Lung cancer screening: review and performance comparison under different risk scenarios. Lung 2014;192:55-63.

58. Tammemägi MC, Church TR, Hocking WG, et al. Evaluation of the lung cancer risks at which to screen everand never-smokers: screening rules applied to the PLCO and NLST cohorts. PLoS Med 2014;11:e1001764.

59. Muller DC, Johansson M, Brennan P. Lung cancer risk prediction model incorporating lung function: development and validation in the UK biobank prospective cohort study. J Clin Oncol 2017;35:861-9.

60. Markaki M, Tsamardinos I, Langhammer A, et al. A validated clinical risk prediction model for lung cancer in smokers of all ages and exposure types: a HUNT study. EBioMedicine 2018;31:36-46.

61. Tammemägi MC, Katki HA, Hocking WG, et al. Selection criteria for lung-cancer screening. N Engl J Med 2013;368:728-36.

62. Kovalchik SA, Tammemagi M, Berg CD, et al. Targeting of low-dose CT screening according to the risk of lungcancer death. N Engl J Med 2013;369:245-54.

63. Bach PB, Kattan MW, Thornquist MD, et al. Variations in lung cancer risk among smokers. J Natl Cancer Inst 2003;95:470-8.

64. Spitz MR, Hong WK, Amos CI, et al. A risk model for prediction of lung cancer. J Natl Cancer Inst 2007;99:715-26.

65. Licaj I, Jacobsen BK, Selmer RM, et al. Smoking and risk of ovarian cancer by histological subtypes: an analysis among 300000 Norwegian women. Br J Cancer 2017;116:270-6.

66. Etzel CJ, Kachroo S, Liu M, et al. Development and validation of a lung cancer risk prediction model for AfricanAmericans. Cancer Prev Res (Phila) 2008;1:255-65.

67. Young RP, Hopkins RJ, Hay BA, et al. A gene-based risk score for lung cancer susceptibility in smokers and exsmokers. Postgrad Med J 2009;85:515-24.

68. Katki HA, Kovalchik SA, Berg CD, et al. Development and validation of risk models to select ever-smokers for CT lung cancer screening. JAMA 2016;315:2300-11.

69. Raji OY, Agbaje OF, Duffy SW, et al. Incorporation of a genetic factor into an epidemiologic model for prediction of individual risk of lung cancer: the Liverpool Lung Project. Cancer Prev Res (Phila) 2010;3:664-9.

70. Hippisley-Cox J, Coupland C. Identifying patients with suspected lung cancer in primary care: derivation and validation of an algorithm. Br J Gen Pract 2011;61:e715-23. 
71. Tammemagi CM, Pinsky PF, Caporaso NE, et al. Lung cancer risk prediction: Prostate, Lung, Colorectal And Ovarian Cancer Screening Trial models and validation. J Natl Cancer Inst 2011;103:1058-68.

72. Hoggart C, Brennan P, Tjonneland A, et al. A risk model for lung cancer incidence. Cancer Prev Res (Phila) 2012;5:834-46.

73. Iyen-Omofoman B, Tata LJ, Baldwin DR, et al. Using socio-demographic and early clinical features in general practice to identify people with lung cancer earlier. Thorax 2013;68:451-9.

74. Park S, Nam BH, Yang HR, et al. Individualized risk prediction model for lung cancer in Korean men. PLoS One 2013;8:e54823.

75. El-Zein RA, Lopez MS, D'Amelio AM, et al. The cytokinesis-blocked micronucleus assay as a strong predictor of lung cancer: extension of a lung cancer risk prediction model. Cancer Epidemiol Biomarkers Prev 2014;23:2462-70.

76. Wilson DO, Weissfeld J. A simple model for predicting lung cancer occurrence in a lung cancer screening program: the Pittsburgh Predictor. Lung Cancer 2015;89:31-7.

77. Kovalchik SA, De Matteis S, Landi MT, et al. A regression model for risk difference estimation in population-based case-control studies clarifies gender differences in lung cancer risk of smokers and never smokers. BMC Med Res Methodol 2013;13:143.

78. Baldwin DR, Duffy SW, Wald NJ, et al. UK Lung Screen (UKLS) nodule management protocol: modelling of a single screen randomised controlled trial of low-dose CT screening for lung cancer. Thorax 2011;66:308-13.

79. Ghimire B, Maroni R, Vulkan D, et al. Evaluation of a health service adopting proactive approach to reduce high risk of lung cancer: the Liverpool Healthy Lung Programme. Lung Cancer 2019;134:66-71.

80. Pastorino U, Boeri M, Sestini S, et al. PL02.04 Blood microRNA and LDCT reduce unnecessary LDCT repeats in lung cancer screening: results of prospective BioMILD trial. J Thorac Oncol 2019;14:S5-6.

81. Mets OM, de Jong PA, Prokop M. Computed tomographic screening for lung cancer: an opportunity to evaluate other diseases. JAMA 2012;308:1433-4.

82. Cordier JF, Cottin V, Khouatra C, et al. Screening for lung cancer and idiopathic pulmonary fibrosis: killing two birds with one stone. Radiology 2014;270:630-1.

83. Molina-Molina M, Aburto M, Acosta O, et al. Importance of early diagnosis and treatment in idiopathic pulmonary fibrosis. Expert Rev Respir Med 2018;12:537-9.

84. Greenland P, LaBree L, Azen SP, et al. Coronary artery calcium score combined with Framingham score for risk prediction in asymptomatic individuals. JAMA 2004;291:210-5.

85. Montalescot G, Sechtem U, Achenbach S, et al. 2013 ESC guidelines on the management of stable coronary artery disease: the Task Force on the management of stable coronary artery disease of the European Society of Cardiology. Eur Heart J 2013;34:2949-3003. Erratum in: Eur Heart J 2014;35:2260-1.

86. Hecht HS, Cronin P, Blaha MJ, et al. 2016 SCCT/ STR guidelines for coronary artery calcium scoring of noncontrast noncardiac chest CT scans: a report of the Society of Cardiovascular Computed Tomography and Society of Thoracic Radiology. J Cardiovasc Comput Tomogr 2017;11:74-84.

87. QRISK®2. Available online: https://www.qrisk.org/2017/

88. Ruparel M, Quaife SL, Dickson JL, et al. Evaluation of cardiovascular risk in a lung cancer screening cohort. Thorax 2019;74:1140-6.

89. Kamel MK, Lee B, Harrison S, et al. Do the surgical results in the National Lung Screening Trial reflect modern thoracic surgical practice? J Thorac Cardiovasc Surg 2019;157:2038-46.e1.

90. Petersen RH, Hansen HJ, Dirksen A, et al. Lung cancer screening and video-assisted thoracic surgery. J Thorac Oncol 2012;7:1026-31.

91. Crestanello JA, Allen MS, Jett JR, et al. Thoracic surgical operations in patients enrolled in a computed tomographic screening trial. J Thorac Cardiovasc Surg 2004;128:254-9.

92. Infante M, Chiesa G, Solomon D, et al. Surgical procedures in the DANTE trial, a randomized study of lung cancer early detection with spiral computed tomography: comparative analysis in the screening and control arm. J Thorac Oncol 2011;6:327-35.

93. Oda R, Okuda K, Osaga S, et al. Long-term outcomes of video-assisted thoracoscopic surgery lobectomy vs. thoracotomy lobectomy for stage IA non-small cell lung cancer. Surg Today 2019;49:369-77.

94. Whitson BA, Andrade RS, Boettcher A, et al. Videoassisted thoracoscopic surgery is more favorable than thoracotomy for resection of clinical stage I non-small cell lung cancer. Ann Thorac Surg 2007;83:1965-70.

95. Murakawa T, Ichinose J, Hino H, et al. Long-term outcomes of open and video-assisted thoracoscopic lung lobectomy for the treatment of early stage non-small cell lung cancer are similar: a propensity-matched study. World 
J Surg 2015;39:1084-91.

96. Bendixen M, Jørgensen OD, Kronborg C, et al. Postoperative pain and quality of life after lobectomy via video-assisted thoracoscopic surgery or anterolateral thoracotomy for early stage lung cancer: a randomised controlled trial. Lancet Oncol 2016;17:836-44.

97. Emmert A, Straube C, Buentzel J, et al. Robotic versus thoracoscopic lung resection: a systematic review and meta-analysis. Medicine (Baltimore) 2017;96:e7633.

98. Reddy RM, Gorrepati ML, Oh DS, et al. Roboticassisted versus thoracoscopic lobectomy outcomes from high-volume thoracic surgeons. Ann Thorac Surg 2018;106:902-8.

99. Pardolesi A, Veronesi G. Robot-assisted lung anatomic segmentectomy. Thorac Surg Clin 2014;24:163-8.

100.Pardolesi A, Park B, Petrella F, et al. Robotic anatomic segmentectomy of the lung: technical aspects and initial results. Ann Thorac Surg 2012;94:929-34.

101. Toker A, Ayalp K, Uyumaz E, et al. Robotic lung segmentectomy for malignant and benign lesions. J Thorac Dis 2014;6:937-42.

102. Cerfolio RJ, Watson C, Minnich DJ, et al. One hundred planned robotic segmentectomies: early results, technical details, and preferred port placement. Ann Thorac Surg 2016;101:1089-95; discussion 1095-6.

103. Geraci TC, Ferrari-Light D, Kent A, et al. Technique, outcomes with navigational bronchoscopy using indocyanine green for robotic segmentectomy. Ann Thorac Surg 2019;108:363-9.

104.Pardolesi A, Veronesi G, Solli P, et al. Use of indocyanine green to facilitate intersegmental plane identification during robotic anatomic segmentectomy. J Thorac Cardiovasc Surg 2014;148:737-8.

105. Callister MEJ, Baldwin DR, Akram AR, et al. British Thoracic Society guidelines for the investigation and management of pulmonary nodules. Thorax 2015;70 Suppl 2:ii1-54.

106. MacMahon H, Naidich DP, Goo JM, et al. Guidelines for management of incidental pulmonary nodules detected on CT images: from the Fleischner Society 2017. Radiology 2017;284:228-43.

107.LeCun Y, Bengio Y, Hinton G. Deep learning. Nature 2015;521:436-44.

108. Baldwin DR, Gustafson J, Pickup L, et al. External validation of a convolutional neural network artificial intelligence tool to predict malignancy in pulmonary nodules. Thorax 2020;75:306-12.

109.Siravegna G, Marsoni S, Siena S, et al. Integrating liquid biopsies into the management of cancer. Nat Rev Clin Oncol 2017;14:531-48.

110.Inamura K. Diagnostic and therapeutic potential of microRNAs in lung cancer. Cancers (Basel) 2017;9:49.

111. Guibert N, Pradines A, Favre G, et al. Current and future applications of liquid biopsy in nonsmall cell lung cancer from early to advanced stages. Eur Respir Rev 2020;29:190052.

112.Ilie M, Hofman V, Long-Mira E, et al. "Sentinel" circulating tumor cells allow early diagnosis of lung cancer in patients with chronic obstructive pulmonary disease. PLoS One 2014;9:e111597.
Cite this article as: Novellis $\mathrm{P}$, Cominesi SR, Rossetti F, Mondoni M, Gregorc V, Veronesi G. Lung cancer screening: who pays? Who receives? The European perspectives. Transl Lung Cancer Res 2021;10(5):2395-2406. doi: 10.21037/tlcr20-677 